\title{
Lower Mean Corpuscular Hemoglobin Concentration is Associated with Favorable Prognosis of Resected Pancreatic Cancer.
}

\section{Dan Wang}

Harbin Medical University

\section{Xiaona Fan}

Harbin Medical University

\section{Lin Fang}

Harbin Medical University

\section{Tianshuo Zhou}

Fifth Affiliated Hospital of Sun Yat-sen University

\section{Qingwei Li}

Harbin Medical University

\section{Wenjing Zhang}

Harbin Medical University

Jinshuang Liu

Harbin Medical University

\section{Ruiyang Li}

Harbin Medical University

Shuling Han

Harbin Medical University

Zhiwei Li ( $\square$ Izhw0451@163.com )

Harbin Medical University

\section{Research Article}

Keywords: adical pancreatomy, pancreatic cancer, mean corpuscular hemoglobin concentration, overall survival

Posted Date: December 3rd, 2020

DOl: https://doi.org/10.21203/rs.3.rs-109365/v1

License: (c) (i) This work is licensed under a Creative Commons Attribution 4.0 International License. 

Lower mean corpuscular hemoglobin concentration is associated with favorable prognosis of resected pancreatic cancer.

Dan Wang ${ }^{1, \#}$, Xiaona Fan ${ }^{1, \#}$, Lin Fang ${ }^{1}$, Tianshuo Zhou ${ }^{2}$, Qingwei Li $^{1}$, Wenjing Zhang ${ }^{1}$, Jinshuang Liu ${ }^{1}$, Ruiyang $\mathrm{Li}^{1}$, Shuling $\mathrm{Han}^{1}$, Zhiwei $\mathrm{Li}^{1,}{ }^{*}$

\# Dan Wang, and Xiaona Fan contributed equally to this work.

*Corresponding author:

ZhiWei Li, MD./Ph.D. Professor, Department of Gastrointestinal Medical Oncology, Harbin Medical University Cancer Hospital, 150 Haping Road, Harbin, Heilongjiang Province 150001, P. R. China. Email: 1zhw0451@163.com;

Tel/Fax: 86-0451-86298278

1Department of Gastrointestinal Medical Oncology, Harbin Medical University Cancer Hospital, 150 Haping Road, Harbin, Heilongjiang Province 150001, P. R. China.

2Cancer Center of the Fifth Hospital of Sun Yat-sen University, 52 Meihua East Road, Zhuhai City, Guangzhou 519000, China.

\begin{tabular}{lll}
\hline Name & indication & e-mail \\
\hline Dan Wang & First author & wd521213@163.com \\
Xiaona Fan & First author & 1290068051@qq.com \\
Lin Fang & Second author & 15910152625@163.com \\
Tianshuo Zhou & Third author & Woshizts@hotmail.com \\
Qingwei Li & Forth author & leldog@163.com \\
Wenjing Zhang & Fifth author & 2384009500@qq.com \\
Jinshuang Liu & Sixth author & 1779116465@qq.com \\
Ruiyang Li & Seventh author & 870467797@qq.com \\
Shuling Han & eighth author & Xixida163@163.com \\
Zhiwei Li & Corresponding Author & Lzhw0451@163.com \\
\hline
\end{tabular}




\section{Abstract}

Objective: This study was designed to explore the value of mean corpuscular hemoglobin concentration (MCHC) for overall survival (OS) in resectable pancreatic cancer (PC).

Background: Systemic inflammatory response are important factors that promotes the occurrence and metastasis of malignant tumors. MCHC are suggested to be relevant to the prognosis of several malignancies. Such as lung cancer, head and neck cancers. However, there are few studies to explore the correlation between them and PC.

\section{Methods:}

The present study included totally 544 patients with PC who underwent radical resection between March 2011 and May 2019. These patients were classified into a training set $(n=195)$ and a validation set $(n=349)$. Kaplan-Meier curve method and Cox proportional hazard regression model were employed to analyze prognosis.

\section{Results:}

Survival curve showed that OS were significantly favorable for resectable PC patients with low preoperative MCHC $(\mathrm{P}=0.015)$. Multivariate analysis suggested that MCHC (hazard ratio [HR], 1.266; 95\% confidential interval $[\mathrm{CI}], 0.895-1.792 ; \mathrm{P}=0.028$ ) were independent prognostic factors for resectable PC patients.

\section{Conclusion:}

Low MCHC was positively correlated with OS in resectable PC, which could be used to predict the prognosis.

Keywords: radical pancreatomy; pancreatic cancer; mean corpuscular hemoglobin concentration; overall survival; 


\section{Introduction}

At present, pancreatic cancer (PC) is the most malignant tumor of the digestive system. According to the statistics, it is the third cancer-related death in the United States, and possibly turns into the second cancer-related death in the United States by 2030[1]. The median survival time for advanced pancreatic cancer is less than 6 months, and the overall 5-year survival rate is less than $6 \%$ [1]. Surgical resection still remains the only way to cure pancreatic cancer [2]. However, the survival of patients with pancreatic cancer has not been improved significantly. Besides, 5 -year survival rate is inferior to $20 \%$ [3]. Therefore, it is of great significance to find an effective prognostic index to provide personalized treatment for patients who underwent radical pancreatomy.

Inflammatory biomarkers have become the research hotspots, a large number of studies have been conducted globally. Erythrocyte-related serological markers also belong to part of inflammatory biomarkers, and erythrocyte-related inflammatory biomarkers have been shown to be associated with prognosis and recurrence in various different cancers. RDW is a hot research topic of erythrocyte-related inflammatory factors in recent years. The study found that RDW was linked to the prognosis of many malignancies, including gastric cancer [5], blood cancer [6] and kidney cancer [7]. MCHC was found to be associated with prognosis of oral cavity and lung cancer [8], especially related to the onset of prostate cancer [9]. However, few studies have explored the relationship between erythrocyte-related serological markers and pancreatic cancer. Therefore, we conducted this retrospective analysis with the aim to explore the relationship between both of them.

\section{Material and methods}

\section{Patients}

A retrospective review on maintained database was performed as much as possible to identify all patients underwent radical pancreatomy at Harbin Medical University Cancer Hospital from March 2011 to May 2019. Exclusion criteria were as follows: (1) patients who have not underwent radical pancreatomy, (2) patients who have not underwent routine blood examination before surgery, (3) patients with metastatic disease, (4) patients received any other treatment before enrollment (5) patients who have underwent malignant disease in other organs, and (6) patients who had incomplete/inaccurate medical records. 
Patients with burns, severe vomiting, diarrhea, chronic carbon monoxide poisoning, cardiac compensatory dysfunction, and hereditary balloon disease affecting MCHC outcomes were excluded [10]. All included patients $(n=544)$ were assigned to either a training set $(n=195)$ or a validation set $(n=349)$.

\section{Data collection}

Clinicopathologic information was obtained from the medical data platform of the Harbin Medical University Cancer Hospital by two independent investigators. During this procedure, the two independent investigators consulted the medical data platform to resolve any possible bias. These data included the following clinicopathologic information: age, sex, body mass index (BMI), hemoglobin (HBG), mean corpsular hemoglobin (MCH), mean corpuscular hemoglobin concentration (MCHC), red cell distribution width (RDW), carcinoma-embryonic antigen (CEA). The following clinical information was also collected: date of surgery, tumor location, tumor size, stage, differentiation grade, nerve invasion, vascular invasion, pathological staging. The definition of resectable PC referred to the criteria defining resectability status of NCCN Clinical Practice Guidelines in Oncology (3th-edition 2019). All patients with resectable PC underwent R0 surgery and had TNM staging based on postoperative pathology. The TNM stage mentioned referred to the Joint Committee on Cancer (8th Edition).

\section{Blood sample collection and measurements}

Blood samples for routine blood examination were collected via standard venipuncture of the veins in the antecubital fossae (anterior elbow veins). Routine hematology tests, including HBG, MCH, MCHC, RDW, CEA, CA199. $2 \mathrm{ml}$ EDTA anticoagulated peripheral blood and $2 \mathrm{ml}$ plasma anticoagulated by sodium citrate as well as serum samples were obtained before breakfast within 7 days prior to surgery. The samples were promptly centrifuged and processed within two hours. The cell counting of peripheral blood was measured using the SYSMEX XN-9000 full-automated hematology analyzer (Sysmex, Tokyo, Japan).

\section{Follow-up evaluation}

During a mean longitudinal follow-up period of 67 months (from a range of 22 to 103 months) after the 
surgery, 186 deaths events occurred (58.83\%). Patients were followed every three months until death or the end of the study. The final follow-up evaluation was performed in November 2019. The follow-up data included date of primary resection, and date of death. Most follow-up includes physical examination, laboratory tests (blood routine, blood biochemistry, tumor marker CEA), and imaging studies. The primary end point OS was defined as the time from surgery to death from any cause, the latter was defined as the date of last live follow-up during this study (cut-off Nov 2019)

\section{Statistical methods}

Descriptive statistical methods were used to analyze the epidemiological, pathological, and hematological characteristics of the patients. Survival curves were drawn according to the Kaplan-Meier method, survival comparision was executed by Log-rank test. Cox proportional hazard regression model was employed for univariate and multivariate analysis. Statistically significant variables $(P<0.05)$ found in univariate analysis were entered into the Cox regression multivariate model using the backward condition method. All tests were double-sided, and statistical significance was inferred at a $\mathrm{P}$ value of 0.05. All statistical analysis was accomplished using SPSS for Windows version25.

\section{RESULTS}

\section{Clinical features of patients}

Of the 544 PC patients included in the study, 195 patients were assigned to the training group and 349 patients were assigned to the validation set. The training set detailed baseline characteristics of the resectable PC patients were shown in Table 1A. The median follow-up time was 762 days. and 95 patients died during follow-up. 114(58.46\%) were male. In 132(67.69\%) patients, the tumor was located in the head of the pancreas. According to the TNM stage, the majority of patients $112(57.44 \%)$ were classified as stage II. The baseline level of the red blood cell correlation index in the training set is shown in Table 1A. The clinicopathological features of resectable PC patients in the validation set are shown in Table 1B. As shown in Table 1B, a total of 349 patients. The median follow-up time was 661 days, and 223 patients died during follow-up. 209(59.89\%) were male. Moderately differentiated people account for the vast majority 200(57.31\%). Patients with neurological invasion account for about 102(29.23\%). The 
baseline level of the red blood cell correlation index in the validation set is shown in Table $1 \mathrm{~B}$. Postoperative adjuvant therapy modalities in 149 (27.4\%) patients included chemotherapy, radiotherapy, chemotherapy. The postoperative adjuvant therapy modalities administered to patients are summarized in Table 2.

\section{The optimal cut-off value of HBG, MCH, MCHC, RDW, CEA for survival analysis.}

The ROC curves were used to calculate the best cut-off values of HBG, MCH, MCHC, RDW and CEA. The optimum cut-off value of preoperative $\mathrm{HBG}, \mathrm{MCH}, \mathrm{MCHC}$, RDW and CEA of resectable PC patients in the training set were $135.6,31.45,340.5,13.75$ and 4.255 , respectively. According to the optimum cut-off value, 195 patients with resectable PC were divided into low-value group and highvalue group (Table 1A). The detailed data of ROC curve analysis are shown in Table 3 and Figure 1.

\section{MCHC and $\mathrm{MCH}$ were associated with prognosis of resectable PC}

The Kaplan-Meier survival curves indicated that patients in low $\mathrm{MCHC}(\mathrm{p}<0.001)$ and low $\mathrm{MCH}$ $(p=0.026)$ groups had longer OS than those in the respective high-value groups (Figure. 2A-B). We verified this conclusion in the validation set as well (Figure. 3A-B). Although Low HBG(p=0.029), $\mathrm{RDW}(\mathrm{p}=0.014)$ and CEA $(\mathrm{p}=0.013)$ were associated with greater OS (Figure. $2 \mathrm{C}-\mathrm{E})$ in training set, we did not confirm his effectiveness in the validation set (Figure. 3C-E).

\section{MCHC was an independent prognostic factor for resectable PC.}

The Schoenfeld residual method was used to determine the Proportional hazards assumption, referred to as the PH assumption. The basic assumption of the $\mathrm{PH}$ hypothesis is: the influence of covariates on survival rate do not change with time, the hazard ratio $\mathrm{h}(\mathrm{t}) / \mathrm{h} 0(\mathrm{t})$ is a fixed value. The results of the Cox regression model are meaningful only when the conditions of the $\mathrm{PH}$ assumption are met. The results showed that there was no correlation between the Schoenfeld residuals of the parameters involved in this study and the rank of failure time, which suggested that the hazard ratios of all covariates would not increase with time. It could be considered that the $\mathrm{PH}$ assumption was satisfied and appropriate Perform 
Cox regression analysis directly. The specific details were shown in Figure 4.

Univariate analysis revealed that Nerve invasion (HR, 0.183; 95\%, 0.074 to $0.451 ; p=0.001$ ), Postoperative adjuvant therapy $(H R, 1.576 ; 95 \%, 1.045$ to $2.375 ; p=0.030), \quad M C H(H R, 1.587 ; 95 \%$ $\mathrm{Cl}, 1.054$ to 2.389; $\mathrm{p}=0.027)$, $\mathrm{MCHC}(\mathrm{HR}, 2.692 ; 95 \% \mathrm{Cl}, 1.723$ to 4.207; $p<0.001)$, RDW (HR, 1.722; 95\% Cl, 1.107 to 2.676; $\mathrm{p}=0.016$ ) , and CEA ( $\mathrm{HR}, 1.292 ; 95 \% \mathrm{Cl}, 0.985$ to $1.695 ; \mathrm{p}=0.014)$ were significantly prognostic factors for resectable PC. In addition, multivariate analyzes showed Nerve invasion (HR, $0.214 ; 95 \%$ CI, 0.087 to $0.529 ; \mathrm{p}=0.001)$, Postoperative adjuvant therapy (HR, $0.976 ; 95 \%$ CI, 0.626 to $1.522 ; \mathrm{p}=0.041)$, RDW (HR, $1.459 ; 95 \%$ CI, 0.928 to $2.293 ; \mathrm{p}=0.012)$,CEA (HR, $1.533 ; 95 \%$ CI, 1.022 to $2.299 ; \mathrm{p}=0.039)$, and $\mathrm{MCHC}(\mathrm{HR}, 2.253 ; 95 \% \mathrm{CI}, 1.380$ to $3.677 ; \mathrm{p}=0.001)$ were independent prognostic factors for resectable PC (Table 4).

\section{DISCUSSION}

The evidence indicated that tumor-associated inflammation and tumor microenvironment play a more and more important role in the cancer development, progression, metastasis [11], and clinical prognosis [12]. As reported previously, MCHC were closely related to the prognosis in several types of cancers. In the present study, it could also be observed that low MCHC can indicate better OS. Consequently, it is reasonable to combine $\mathrm{MCHC}$ as a simple and convenient marker so as to enrich the stratification of prognosis in PC patients.

MCHC means that the average red blood cell hemoglobin concentration is calculated by dividing the red blood cell specific volume (HCT) by the average red blood cell volume (MCV), which is an indicator of the average red blood cell volume [13]. MCHC indirectly reflects the degree of anemia from a quantitative perspective. Admittedly, anemia was associated with poor survival rates for a variety of malignancies. Qu found that low MCHC (<335) was linked to unfavorable prognosis of resected lung cancer [7]. Additionally, studies also showed that MCHC was associated with prognosis for oral and head and neck cancers. Based on the obtained findings, preoperative $\mathrm{MCHC}$ was an independent predictor of OS for pancreatic cancer patients undergoing curative surgical resection. To date, only a few preliminary hypotheses explain why MCHC may affect prognosis. Nevertheless, the mechanism does not remain clear. This may be related to the following mechanism. At first, Hemoglobin is the main driving force for carrying iron, and anemia can lead to excessive iron load. Iron is a trace element involved in a wide 
range of human metabolism. Iron deficiency and excess can lead to disease. Iron deficiency has been linked to anemia [14], chronic heart failure [15] as well as other conditions. Excess iron increases the risk of cancer [16]. Even if the excess is physiological, the risk of cancer can still increase[17]. In other words, proper iron consumption achieves a protective effect on the body. The correlation between hematological parameters and serum iron markers may be attributed to changes in iron levels that are reflected in erythrocyte morphology to some extent [18]. Second, iron overload often accelerates and induces oxidative stress. Oxidative stress is another factor that causes cancer to develop, and serum iron can induce and accelerate the current process [19]. Furthermore, the changes of red blood cell parameters indirectly reflect the process of oxidative stress. Third, Hemoglobin is the main driving force for carrying oxygen, and low hemoglobin can cause tissue hypoxia. Hypoxia can promote tumor progression and induce changes in the cell genome to enhance tumor growth and angiogenesis. Hypoxia induces epithelial mesenchyme transformation (EMT) [20], which is critical for cancer progression and metastasis. In addition, hypoxia also affects angiogenesis [21]. On the conditions of hypoxia, cancer cells exhibit adaptive metabolic changes. These include the conversion of glucose to lactic acid and increased glucose uptake through promotive glucose transporters (GLUTs), which is a phenomenon also known as the Warburg effect [22].Finally, nutrition is another factor contributing to the development of disease [23]. Reduced hemoglobin indicates that patient's nutritional status is relatively poor. There exists the increased incidence of complications and increased mortality in patients with nutritional risks before surgery [24]. In clinic, accurate pretreatment staging is essential for the treatment decision. Our study found that preoperative $\mathrm{MCHC}$ is an independent prognostic factor for pancreatic cancer, which can affect the prognosis of pancreatic cancer patients. Therefore, as the novel and easily obtainable prognostic marker, preoperative $\mathrm{MCHC}$ can be used as a supplement to pathological stages, which can also provide a more accurate prognosis.

However, the present study also had some flaws. At first, the current work was a retrospective study. Although we had excluded factors such as blood system diseases and inflammatory diseases that might cause MCHC changes before the study, there were still a lot of unavoidable interference factors which could affect the results of this experiment. Then, this study was single-centered with small sample, leading to the biases in sample selection and results analysis. More medical institutions and samples are still needed to further verify the obtained result. In summary, although this study had certain shortcomings, MCHC still had good predictive values for the prognosis of patients with pancreatic cancer 
after surgery. In the meanwhile, these indexes achieved the advantages of cheap, convenient, noninvasive, as well as certain research values.

Declarations

Ethics approval and consent to participate

This retrospective study involving human participants meets the ethical standards of the institution and the National Research Council as well as the 1964 Helsinki Declaration and its subsequent amendments or comparable ethical standards. The Human Investigation Committee (IRB) at Harbin Medical University approved the study.The ethical number is: 2020-083-IIIT. Informed consent was acquired from all individual participators incorporated in the study.

Consent for publication

All authors commented on previous versions of the manuscript. All authors read and approved the final manuscript.

Availability of data and materials

All of the data collected and analysed during the current study are available from the corresponding author on rational demand.

Competing interests

statement: The authors declare that they have no competing interests

Funding

This study was sponsored by the National Natural Science Foundation of China (No.81773210); Haiyan Research Fund (No. JJLX2016-01); And Graduate Research and Practice Innovation Project. Authors' contributions

All authors dedicated to the study notion and design. Material preparation, data collection and analysis were accomplished by DW, XNF and LF. The first draft of the manuscript was written by DW. TSZ, WJZ, JSL, RYL, SLH collected the date. QWL, ZWL revised the work critically for important intellectual content.

Acknowledgements

Not applicable

References:

[1]. Mittler, R., M. Darash-Yahana, Y. S. Sohn, F. Bai, L. Song, I. Z. Cabantchik, P. A. Jennings, J. N. Onuchic and R. Nechushtai . "NEET Proteins: A New Link Between Iron Metabolism, Reactive 
Oxygen Species, and Cancer." Antioxid Redox Signal (2019) 30(8): 1083-1095.

[2]. Rahib, L., B. D. Smith, R. Aizenberg, A. B. Rosenzweig, J. M. Fleshman and L. M. Matrisian . "Projecting cancer incidence and deaths to 2030: the unexpected burden of thyroid, liver, and pancreas cancers in the United States." Cancer Res(2014)74(11): 2913-2921.

[3]. Strobel, O., J. Neoptolemos, D. Jager and M. W. Buchler . "Optimizing the outcomes of pancreatic cancer surgery." Nat Rev Clin Oncol(2019)16(1): 11-26.

[4]. Abe, T., C. Koi, S. Kohi, K. B. Song, K. Tamura, A. Macgregor-Das, N. Kitaoka, M. Chuidian, M. Ford, M. Dbouk, M. Borges, J. He, R. Burkhart, C. L. Wolfgang, A. P. Klein, J. R. Eshleman, R. H. Hruban, M. I. Canto and M. Goggins. "Gene Variants That Affect Levels of Circulating Tumor Markers Increase Identification of Patients with Pancreatic Cancer." Clin Gastroenterol Hepatol.(2019).

[5]. Hirahara, N., Y. Tajima, Y. Fujii, S. Kaji, T. Yamamoto, R. Hyakudomi, T. Taniura and Y. Kawabata . "Comprehensive Analysis of Red Blood Cell Distribution Width as a Preoperative Prognostic Predictor in Gastric Cancer." Anticancer Res (2019)39(6): 3121-3130.

[6]. Ai, L., S. Mu and Y. Hu . "Prognostic role of RDW in hematological malignancies: a systematic review and meta-analysis." Cancer Cell Int (2018)18: 61.

[7]. Zyczkowski, M., P. Rajwa, E. Gabrys, K. Jakubowska, E. Jantos and A. Paradysz. "The Relationship Between Red Cell Distribution Width and Cancer-Specific Survival in Patients With Renal Cell Carcinoma Treated With Partial and Radical Nephrectomy." Clin Genitourin Cancer (2018)16(3): e677-e683.

[8]. Qu, X., T. Zhang, H. Ma, P. Sui and J. J. F. O. Du. "Lower mean corpuscular hemoglobin concentration is associated with unfavorable prognosis of resected lung cancer."(2014)10(14): 2149-2159.

[9]. Adris, N., A. C. G. Chua, M. W. Knuiman, M. L. Divitini, D. Trinder and J. K. Olynyk. "A prospective cohort examination of haematological parameters in relation to cancer death and incidence: the Busselton Health Study." BMC Cancer(2018)18(1): 863.

[10]. Rajizadeh, A., H. Mozaffari-Khosravi, J. Zavar-Reza and S. M. Shiryazdi. "Comparison of hematological parameters, iron levels, and oxidative stress in women with and without breast cancer: A case- control study." Med J Islam Repub Iran(2017)31: 114.

[11]. Chan, J. C., D. L. Chan, C. I. Diakos, A. Engel, N. Pavlakis, A. Gill and S. J. Clarke. "The 
Lymphocyte-to-Monocyte Ratio is a Superior Predictor of Overall Survival in Comparison to Established Biomarkers of Resectable Colorectal Cancer." Ann Surg(2017)265(3): 539-546.

[12]. Gao L , Zhang H , Zhang B , et al. Prognostic value of combination of preoperative platelet count and mean platelet volume in patients with resectable non-small cell lung cancer[J]. Oncotarget, 2017, 8(9).

[13]. Huang, X. Z., Y. C. Yang, Y. Chen, C. C. Wu, R. F. Lin, Z. N. Wang and X. Zhang . "Preoperative Anemia or Low Hemoglobin Predicts Poor Prognosis in Gastric Cancer Patients: A Meta-Analysis." Dis Markers 2019: 7606128.

[14]. Stauder, R., P. Valent and I. Theurl. "Anemia at older age: etiologies, clinical implications, and management." Blood(2018) 131(5): 505-514.

[15]. Yerlikaya, A., M. C. Bulbul, B. Afsar, T. Dagel, G. Aslan, L. Voroneanu, D. Siriopol, A. Covic and M. Kanbay. "Iron in kidney and heart failure: from theory to practice." Int Urol Nephrol(2018) 50(3): $481-493$

[16]. Lagergren, K., K. Wahlin, F. Mattsson, D. Alderson and J. Lagergren. "Haemochromatosis and gastrointestinal cancer." Int J Cancer (2016)139(8): 1740-1743.

[17]. Chua, A. C., M. W. Knuiman, D. Trinder, M. L. Divitini and J. K. Olynyk. "Higher concentrations of serum iron and transferrin saturation but not serum ferritin are associated with cancer outcomes." Am J Clin Nutr(2016) 104(3): 736-742.

[18]. Kautz, L. and E. Nemeth. "Molecular liaisons between erythropoiesis and iron metabolism." Blood(2014) 124(4): 479-482..

[19]. Miller, K. D., L. Nogueira, A. B. Mariotto, J. H. Rowland, K. R. Yabroff, C. M. Alfano, A. Jemal, J. L. Kramer and R. L. Siegel . "Cancer treatment and survivorship statistics, 2019." CA Cancer J Clin (2019)69(5): 363-385

[20].Zhao, B., Y. Mei, J. Yang and P. Ji . "Erythropoietin-regulated oxidative stress negatively affects enucleation during terminal erythropoiesis." Exp Hematol(2016) 44(10): 975-981.

[21]. Feng, Y., K. Song, W. Shang, L. Chen, C. Wang, B. Pang and N. J. O. L. Wang . "REDD1 overexpression in oral squamous cell carcinoma may predict poor prognosis and correlates with high microvessel density.(2019)

[22]. Urata, K., I. Kajihara, H. Miyauchi, T. Mijiddorj, S. Otsuka-Maeda, R. Sakamoto, S. Sawamura, H. Kanemaru, S. Kanazawa-Yamada, K. Makino, J. Aoi, T. Makino, S. Fukushima, Y. Komohara and 
H. Ihn . "The Warburg effect and tumor immune microenvironment in extramammary Paget's disease: Overexpression of LDHA correlates with immune resistance." J Eur Acad Dermatol Venereol(2019).

[23]. Dou, L., X. Wang, Y. Cao, A. Hu and L. Li . "Relationship between Postoperative Recovery and Nutrition Risk Screened by NRS 2002 and Nutrition Support Status in Patients with Gastrointestinal Cancer." Nutr Cancer(2020)72(1): 33-40.

[24]. Peng, H., B. B. Chen, L. L. Tang, L. Chen, W. F. Li, Y. Zhang, Y. P. Mao, Y. Sun, L. Z. Liu, L. Tian, Y. Guo and J. Ma. "Prognostic value of nutritional risk screening 2002 scale in nasopharyngeal carcinoma: A large-scale cohort study." Cancer Sci(2018)109(6): 1909-1919.

[25]. TABLE1 A. Clinical and pathological characteristics of 195 resectable PC patients in training set

\begin{tabular}{|c|c|}
\hline Characteristic & Score (195) \\
\hline \multicolumn{2}{|l|}{ Age } \\
\hline$\leqslant 53.5$ & $54(27.69 \%)$ \\
\hline$>53.5$ & $141(72.31 \%)$ \\
\hline \multicolumn{2}{|l|}{ Gender } \\
\hline Male & $114(58.46 \%)$ \\
\hline Female & $81(41.54 \%)$ \\
\hline \multicolumn{2}{|l|}{ BMI } \\
\hline$\leqslant 27.71$ & $177(90.77 \%)$ \\
\hline$>27.71$ & $18(9.23 \%)$ \\
\hline \multicolumn{2}{|l|}{ Tumor location } \\
\hline Caput pancreatis & $132(67.69 \%)$ \\
\hline Pancreatic body and tail & $63(32.31 \%)$ \\
\hline \multicolumn{2}{|l|}{ Differentiation } \\
\hline Well-differentiated & $24(12.31 \%)$ \\
\hline Moderately differentiated & $100(51.28 \%)$ \\
\hline Poorly differentiated & $71(36.41 \%)$ \\
\hline \multicolumn{2}{|l|}{$\mathrm{T}$} \\
\hline 1 & $29(14.87 \%)$ \\
\hline 2 & $73(37.44 \%)$ \\
\hline 3 & $93(47.69 \%)$ \\
\hline \multicolumn{2}{|l|}{$\mathrm{N}$} \\
\hline 0 & $144(73.85 \%)$ \\
\hline 1 & $44(22.56 \%)$ \\
\hline 2 & $7(3.59 \%)$ \\
\hline \multicolumn{2}{|l|}{ pTNM stage } \\
\hline I & $76(38.97 \%)$ \\
\hline
\end{tabular}


II

III

Vascular invasion

positive

negative

Nerve invasion

positive

negative

HBG

$\leqslant 135.6$

$>135.6$

$\mathrm{MCH}$

$\leqslant 31.45$

$>31.45$

$\mathrm{MCHC}$

$\leqslant 340.5$

$>340.5$

RDW

$\leqslant 13.75$

$>13.75$

CEA

$\leqslant 4.255$

$>4.255$
$112(57.44 \%)$

7(3.59\%)

$8(4.1 \%)$

187 (95.9\%)

$39(20 \%)$

$156(80 \%)$

129(66.15\%)

$66(33.85 \%)$

$100(51.28 \%)$

95(48.72\%)

97(49.74\%)

98(50.26\%)

80(41.03\%)

115(58.97\%)

114 (58.46\%)

81 (41.54\%)

BMI: Body Mass Index MCH: mean corpuscular volume hemoglobin MCHC: mean corpuscular

hemoglobin concerntration HBG: Hemoglobin RDW: Red Cell volume Distribution Width CEA:

carcinoma-embryonic antigen.

TABLE1B. Clinical and pathological characteristics of 349 resectable PC patients in validation set

\begin{tabular}{cc}
\hline Characteristic & Score $(349)$ \\
\hline Age & \\
$\leqslant 53.5$ & $127(36.39 \%)$ \\
$>53.5$ & $222(63.61 \%)$ \\
Gender & \\
Male & $209(59.89 \%)$ \\
Female & $140(40.11 \%)$ \\
BMI & \\
$>27.71$ & $317(90.83 \%)$ \\
$>27.71$ & $32(9.17 \%)$ \\
Tumor location & $129(36.96 \%)$ \\
Caput pancreatis & $220(63.04 \%)$
\end{tabular}




\begin{tabular}{|c|c|}
\hline Differentiation & \\
\hline Well-differentiated & $27(7.74 \%)$ \\
\hline Moderately differentiated & $200(57.31 \%)$ \\
\hline Poorly differentiated & $122(34.96 \%)$ \\
\hline \multicolumn{2}{|l|}{$\mathrm{T}$} \\
\hline 1 & $41(11.75 \%)$ \\
\hline 2 & $106(30.37 \%)$ \\
\hline 3 & $202(57.88 \%)$ \\
\hline \multicolumn{2}{|l|}{$\mathrm{N}$} \\
\hline 0 & $245(70.20 \%)$ \\
\hline 1 & $89(25.50 \%)$ \\
\hline 2 & $15(4.30 \%)$ \\
\hline \multicolumn{2}{|l|}{ pTNM stage } \\
\hline I & $109(31.23 \%)$ \\
\hline II & $224(64.18 \%)$ \\
\hline III & $16(4.58 \%)$ \\
\hline \multicolumn{2}{|l|}{ Vascular invasion } \\
\hline positive & $12(3.44 \%)$ \\
\hline negative & $337(96.56 \%)$ \\
\hline \multicolumn{2}{|l|}{ Nerve invasion } \\
\hline positive & $102(29.23 \%)$ \\
\hline negative & $247(70.77 \%)$ \\
\hline \multicolumn{2}{|l|}{ HBG } \\
\hline$\leqslant 135.6$ & $115(32.95 \%)$ \\
\hline$>135.6$ & $234(67.05 \%)$ \\
\hline \multicolumn{2}{|l|}{$\mathrm{MCH}$} \\
\hline$\leqslant 31.45$ & $130(37.25 \%)$ \\
\hline$>31.45$ & $219(62.75 \%)$ \\
\hline \multicolumn{2}{|l|}{$\mathrm{MCHC}$} \\
\hline$\leqslant 340.5$ & $85(24.36 \%)$ \\
\hline$>340.5$ & $264(75.64 \%)$ \\
\hline \multicolumn{2}{|l|}{ RDW } \\
\hline$\leqslant 13.75$ & $177(50.72 \%)$ \\
\hline$>13.75$ & $172(49.28 \%)$ \\
\hline \multicolumn{2}{|l|}{ CEA } \\
\hline$\leqslant 4.255$ & $243(69.63 \%)$ \\
\hline$>4.255$ & $106(30.37 \%)$ \\
\hline
\end{tabular}

BMI: Body Mass Index MCH: mean corpuscular volume hemoglobin MCHC: mean corpuscular hemoglobin concerntration HBG: Hemoglobin RDW: Red Cell volume Distribution Width CEA: carcinoma-embryonic antigen.

Table 2. Postoperative adjuvant therapy modality for the resectable PC patients

\begin{tabular}{ll}
\hline Therapy modality & No. $(\%)$ \\
\hline Chemotherapy & $138(0.93)$
\end{tabular}




\begin{tabular}{lllll}
\multicolumn{5}{l}{ Radiotherapy } \\
$\begin{array}{l}\text { Table 3. ROC curve results based on } \mathrm{MCHC}, \mathrm{MCH}, \mathrm{RDW} \text {, and HBG of resectable PC patients in } \\
\text { training set }\end{array}$ \\
\hline Biomarker & $\begin{array}{l}\text { Best } \\
\text { cut-off point }\end{array}$ & AUC $(95 \% \mathrm{Cl})$ & $\begin{array}{l}\text { Sensitivity of } \\
\text { Y oden index }\end{array}$ & $\begin{array}{l}\text { Specificity of } \\
\text { Y oden index }\end{array}$ \\
\hline MCHC & 340.50 & $0.750(0.681-0.819)$ & 0.716 & 0.700 \\
MCH & 31.45 & $0.595(0.515-0.675)$ & 0.589 & 0.610 \\
RDW & 13.75 & $0.594(0.514-0.674)$ & 0.705 & 0.520 \\
HBG & 135.60 & $0.507(0.426-0.589)$ & 0.379 & 0.700 \\
CEA & 4.255 & $0.575(0.494-0.655)$ & 0.505 & 0.670 \\
\hline A &
\end{tabular}

AUC, the area under the ROC curve. MCH: mean corpuscular volume hemoglobin MCHC: mean corpuscular hemoglobin concerntration HBG: Hemoglobin RDW: Red Cell volume Distribution Width CEA: carcinoma-embryonic antigen.

TABLE 4. Univariate and multivariate for analyzes of Cox regression model candidate prognostic factors of resectable PC patients

\begin{tabular}{|c|c|c|c|c|}
\hline \multirow[b]{2}{*}{ Characteristics } & \multicolumn{2}{|l|}{ Univariate analysis } & \multicolumn{2}{|l|}{ Multivariate analysis } \\
\hline & HR $(95 \% \mathrm{CI})$ & $\mathrm{P}$ & $\mathrm{HR}(95 \% \mathrm{CI})$ & $\mathrm{P}$ \\
\hline Age & $1.678(1.014-2.779)$ & 0.044 & & \\
\hline Gender & $1.074(0.713-1.618)$ & 0.733 & & \\
\hline BMI & $1.290(0.669-2.486)$ & 0.447 & & \\
\hline Tumor location & $1.426(0.903-2.251)$ & 0.128 & & \\
\hline Differentiation & $1.034(0.757-1.413)$ & 0.823 & & \\
\hline $\mathrm{T}$ & $0.931(0.714-1.215)$ & 0.598 & & \\
\hline $\mathrm{N}$ & $1.121(0.910-1.380)$ & 0.848 & & \\
\hline Stage & $1.886(0.582-6.110)$ & 0.290 & & \\
\hline Vascular invasion & $0.826(0.377-1.806)$ & 0.631 & & \\
\hline Nerve invasion & $0.183(0.074-0.451)$ & $<0.001$ & $0.214(0.087-0.529)$ & 0.001 \\
\hline Postoperative adjuvant therapy & $1.576(1.045-2.375)$ & 0.030 & $0.976(0.626-1.522)$ & 0.041 \\
\hline HBG & $1.395(0.921-2.112)$ & 0.116 & & \\
\hline $\mathrm{MCH}$ & $1.587(1.054-2.389)$ & 0.027 & $1.012(0.646-1.584)$ & 0.960 \\
\hline $\mathrm{MCHC}$ & $2.692(1.723-4.207)$ & $<0.001$ & $2.253(1.380-3.677)$ & 0.001 \\
\hline RDW & $1.722(1.107-2.676)$ & 0.016 & $1.459(0.928-2.293)$ & 0.012 \\
\hline CEA & $1.292(0.985-1.695)$ & 0.014 & $1.533(1.022-2.299)$ & 0.039 \\
\hline
\end{tabular}

PC, pancreatic cancer; HR, hazard ratio; CI, confidence interval; BMI: Body Mass Index HBG:

hemoglobin MCH: mean corpuscular hemoglobin MCHC: mean corpuscular hemoglobin concerntration

RDW: Red Cell volume Distribution Width CEA: carcinoma-embryonic antigen. 

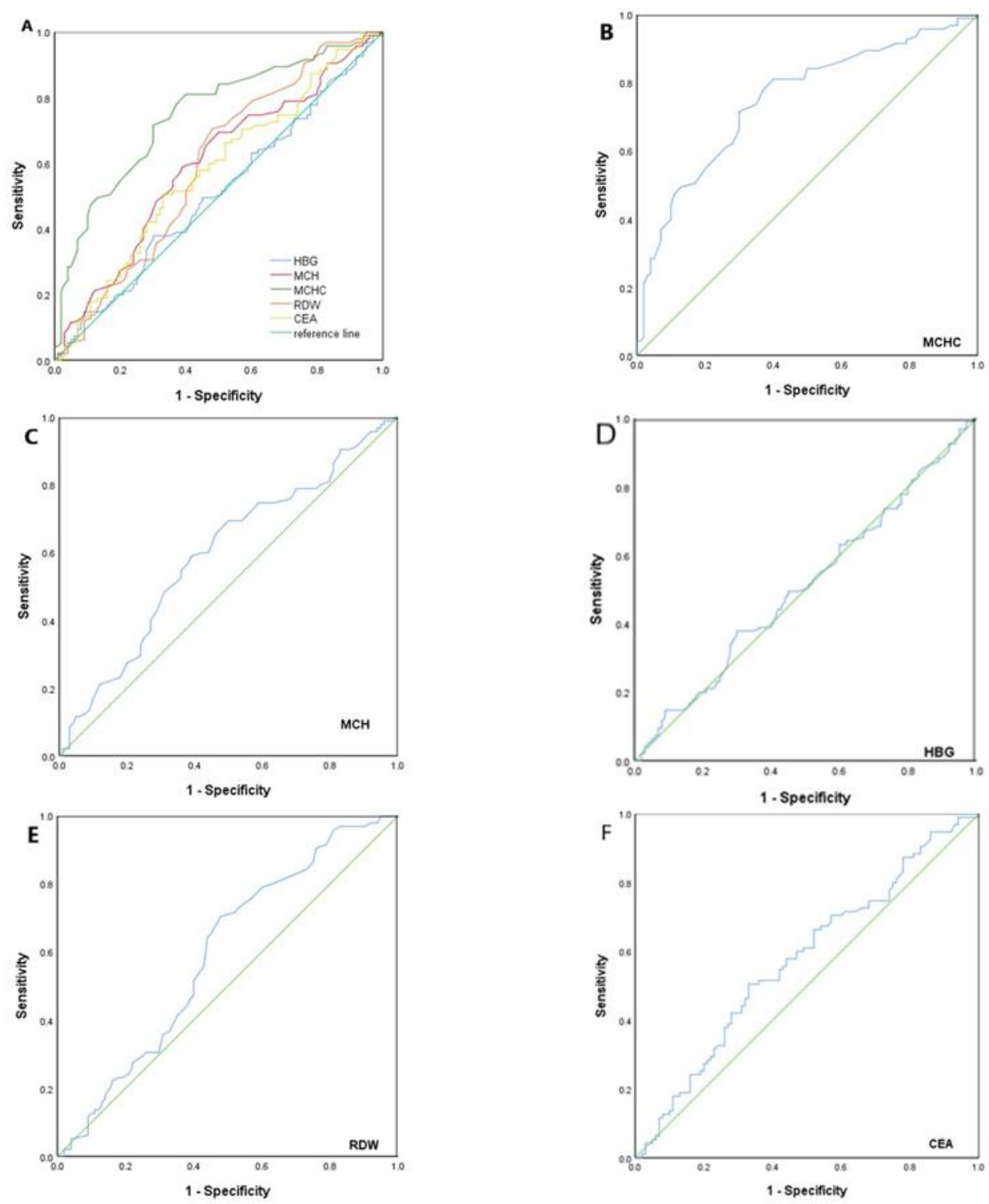

Figure 1. Receiver operating characteristic (ROC) analysis based on mean corpuscular hemoglobin concerntration $(\mathrm{MCHC})(\mathrm{B})$, mean corpuscular volume hemoglobin $(\mathrm{MCH})(\mathrm{C})$, Hemoglobin $(\mathrm{RDW})$ (D), Red Cell volume Distribution Width (RDW) (E), carcinoma-embryonic antigen (CEA)(F) of resectable PC patients in training set. (A) The area under the ROC curve (AUC) indicates the diagnostic power of MCHC was the most powerful. (B) The AUC indicates the diagnostic power of MCHC. In this model, the best cut-off point for MCHC was 340.5, AUC was 0.750 (95\% confidence interval [CI], 0.681 to 0.819 ), the sensitivity of the Yoden index was 0.716 , and the specificity was 0.700 . (C) The AUC indicates the diagnostic power of $\mathrm{MCH}$. In this model, the optimal cut-off point for MCH was 31.35, AUC was 0.595 (95\% CI, 0.515 to 0.675$)$, the sensitivity of the Yoden index was 0.589 , and the specificity was 0.610 . (D) The AUC indicates the diagnostic power of HBG. In this model, the optimal cut-off point for HBG was 135.6, AUC was 0.507 (95\% CI, 0.426 to 0.589 ), the sensitivity of the Yoden 
index was 0.379 , and the specificity was 0.700 . (E) The AUC indicates the diagnostic power of RDW. In this model, the best cut-off point for RDW was 13.75 , AUC was 0.594 (95\% CI, 0.514 to 0.674$)$, the sensitivity of the Yoden index was 0.705 , and the specificity was 0.520 . (F) The AUC indicates the diagnostic power of CEA. In this model, the best cut-off point for CEA was 4.255, AUC was 0.575 (95\% CI, 0.494 to 0.655$)$, the sensitivity of the Yoden index was 0.505 , and the specificity was 0.670 .
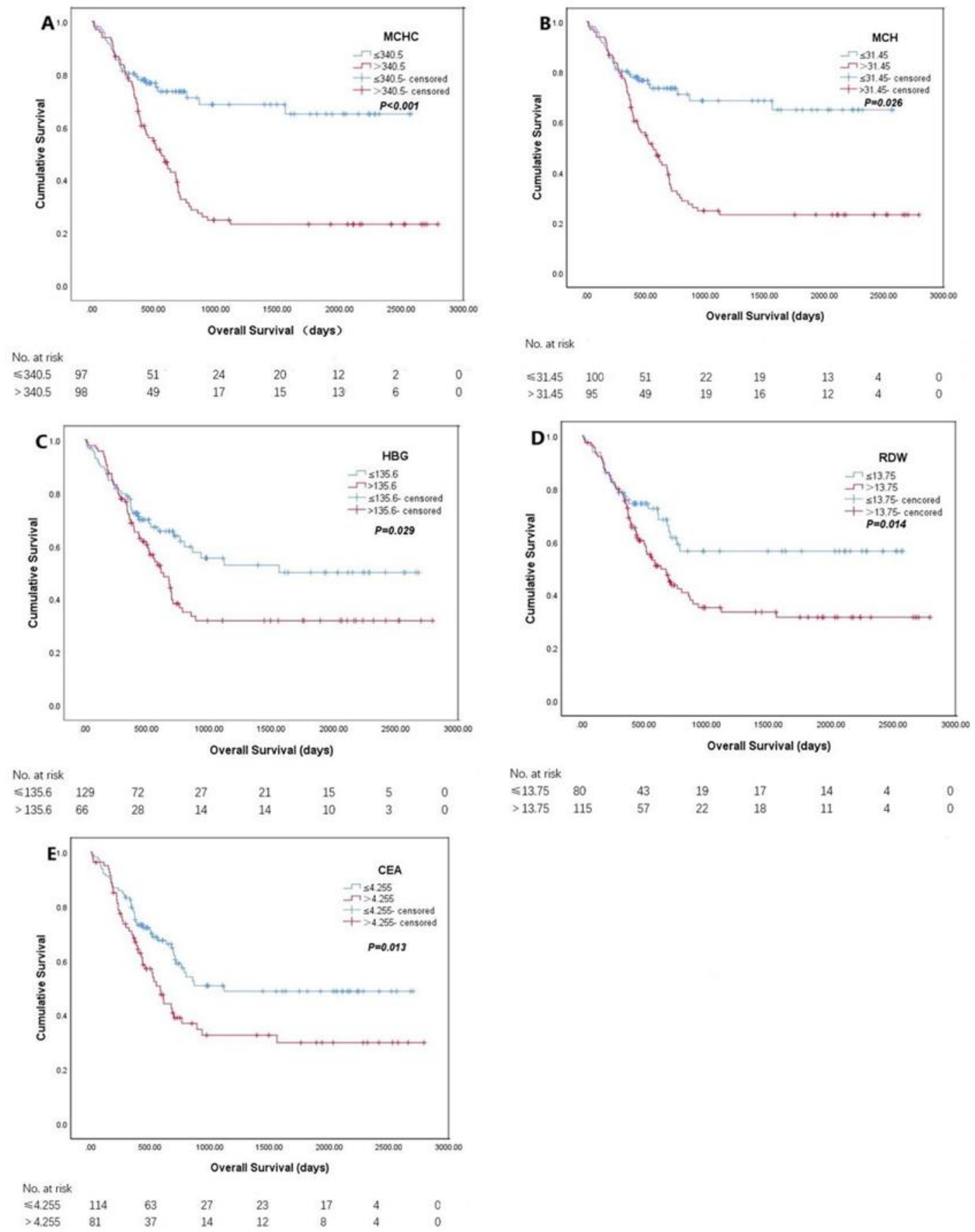

Figure 2. Kaplan-Meier survival curves of resectable PC patients in training set based on $\mathrm{MCHC}, \mathrm{MCH}$, HBG, RDW, CEA. (A) Data in training set compares MCHC $\leqslant 340.5 \mathrm{vs} .>340.5$ (p < 0.05). (B) Data in 
training set compares $\mathrm{MCH} \leqslant 31.45$ vs. $>31.45(\mathrm{p}<0.05)$. (C) Data in training set compares $\mathrm{HBG} \leqslant$ 135.6vs. $>135.6(\mathrm{p}<0.05)$. (D) Data in training set compares RDW $\leqslant 13.75 \mathrm{vs}$. $>13.75(\mathrm{p}<0.05)$. (E) Data in training set compares CEA $\leqslant 4.255 \mathrm{vs} .>4.255(\mathrm{p}<0.05)$.
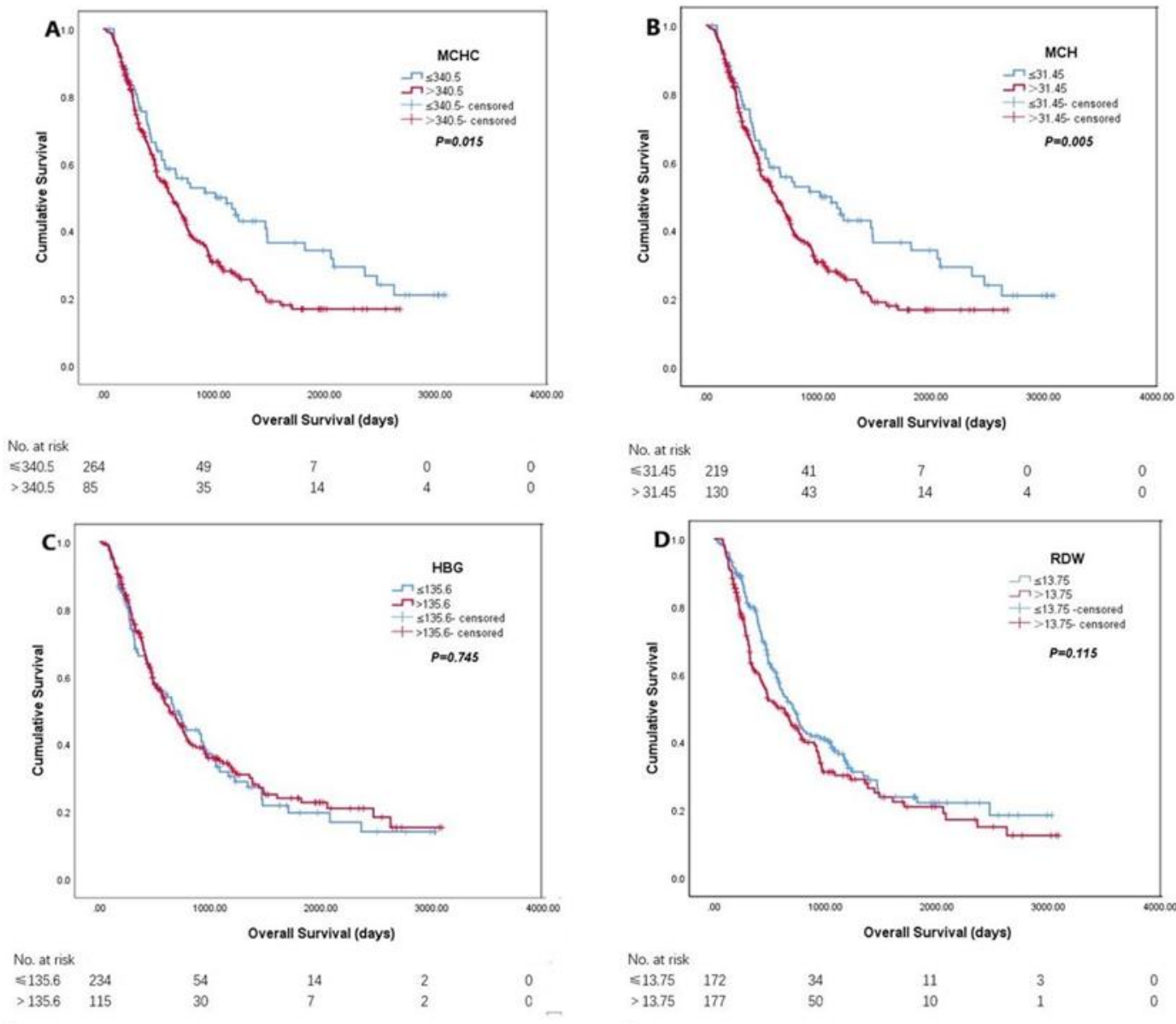

No. at risk
$\leqslant 13.75 \quad 172$
$>1375$

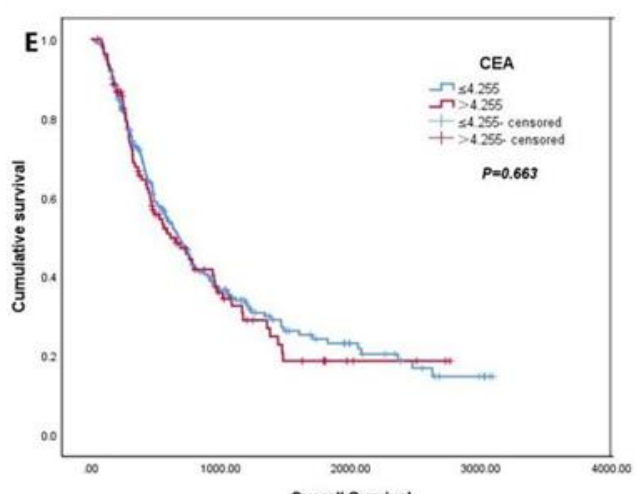

No. at risk

$\leqslant 4.255 \quad 243$

Overall Survival

$\begin{array}{lll}>4.255 & 106 & 23\end{array}$ 
Figure 3. Kaplan-Meier survival curves of resectable PC patients in validation set based on $\mathrm{MCHC}, \mathrm{MCH}$, HBG, RDW, CEA. (A) Data in validation set compares MCHC $\leqslant 340.5 \mathrm{vs} .>340.5$ (p < 0.05). (B) Data in validation set compares $\mathrm{MCH} \leqslant 31.45$ vs. $>31.45(\mathrm{p}<0.05)$. (C) Data in validation set compares $\mathrm{HBG} \leqslant$ 135.6vs. $>135.6(\mathrm{p}>0.05)$. (D) Data in validation set compares $R D W \leqslant 13.75 v s .>13.75(p>0.05)$. (E) Data in validation set compares CEA $\leqslant 4.255 \mathrm{vs} .>4.255(\mathrm{p}>0.05)$.
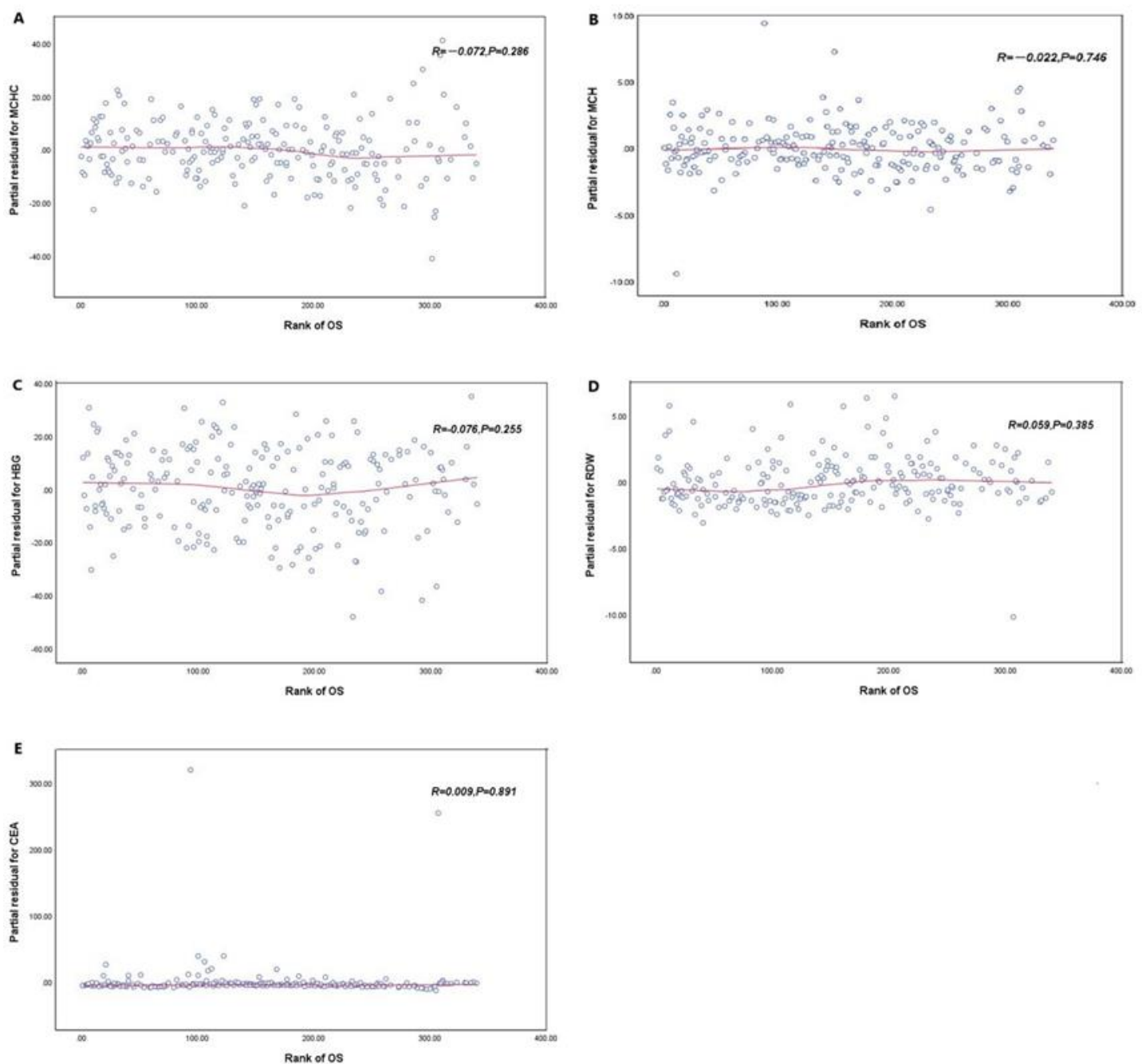

Figure 4. Schoenfeld residual method of resectable PC patients based on $\mathrm{MCHC}, \mathrm{MCH}, \mathrm{HBG}, \mathrm{RDW}$, CEA. (A) Data compares partial residual for $\mathrm{MCHC}$ with rank of $\mathrm{OS}(\mathrm{R}=-0.072, \mathrm{P}>0.05)$. (B) Data compares partial residual for $\mathrm{MCH}$ with rank of $\mathrm{OS}(\mathrm{R}=-0.022, \mathrm{P}>0.05)$. (C) Data compares partial residual for HBG with rank of OS $(\mathrm{R}=-0.076, \mathrm{P}>0.05)$. (D) Data compares partial residual for RDW 
with rank of OS $(\mathrm{R}=0.059, \mathrm{P}>0.05)$. (E) Data compares partial residual for CEA with rank of OS $(\mathrm{R}=$ $-0.009, \mathrm{P}>0.05)$ 
Figures
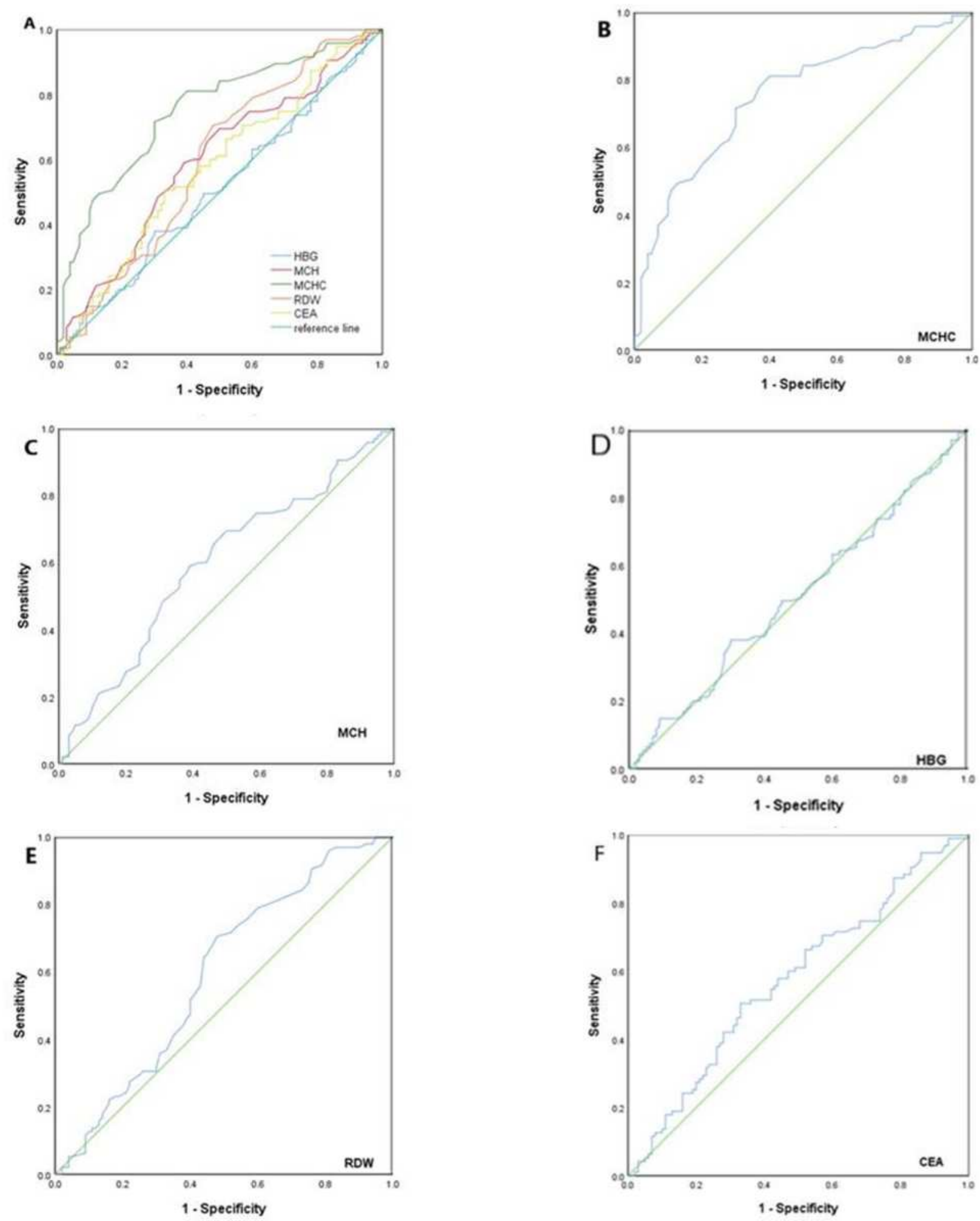

Figure 1

Receiver operating characteristic (ROC) analysis based on mean corpuscular hemoglobin concerntration $(\mathrm{MCHC})(\mathrm{B})$, mean corpuscular volume hemoglobin $(\mathrm{MCH})(\mathrm{C})$, Hemoglobin (RDW) (D), Red Cell volume Distribution Width (RDW) (E), carcinoma-embryonic antigen (CEA)(F) of resectable PC 
patients in training set. (A) The area under the ROC curve (AUC) indicates the diagnostic power of MCHC was the most powerful. (B) The AUC indicates the diagnostic power of MCHC. In this model, the best cutoff point for MCHC was 340.5, AUC was 0.750 (95\% confidence interval [Cl], 0.681 to 0.819 ), the sensitivity of the Yoden index was 0.716 , and the specificity was 0.700 . (C) The AUC indicates the diagnostic power of $\mathrm{MCH}$. In this model, the optimal cut-off point for $\mathrm{MCH}$ was 31.35, AUC was 0.595 ( $95 \% \mathrm{Cl}, 0.515$ to 0.675$)$, the sensitivity of the Yoden index was 0.589 , and the specificity was 0.610 . (D) The AUC indicates the diagnostic power of HBG. In this model, the optimal cut-off point for HBG was 135.6, AUC was $0.507(95 \% \mathrm{Cl}, 0.426$ to 0.589$)$, the sensitivity of the Yoden 

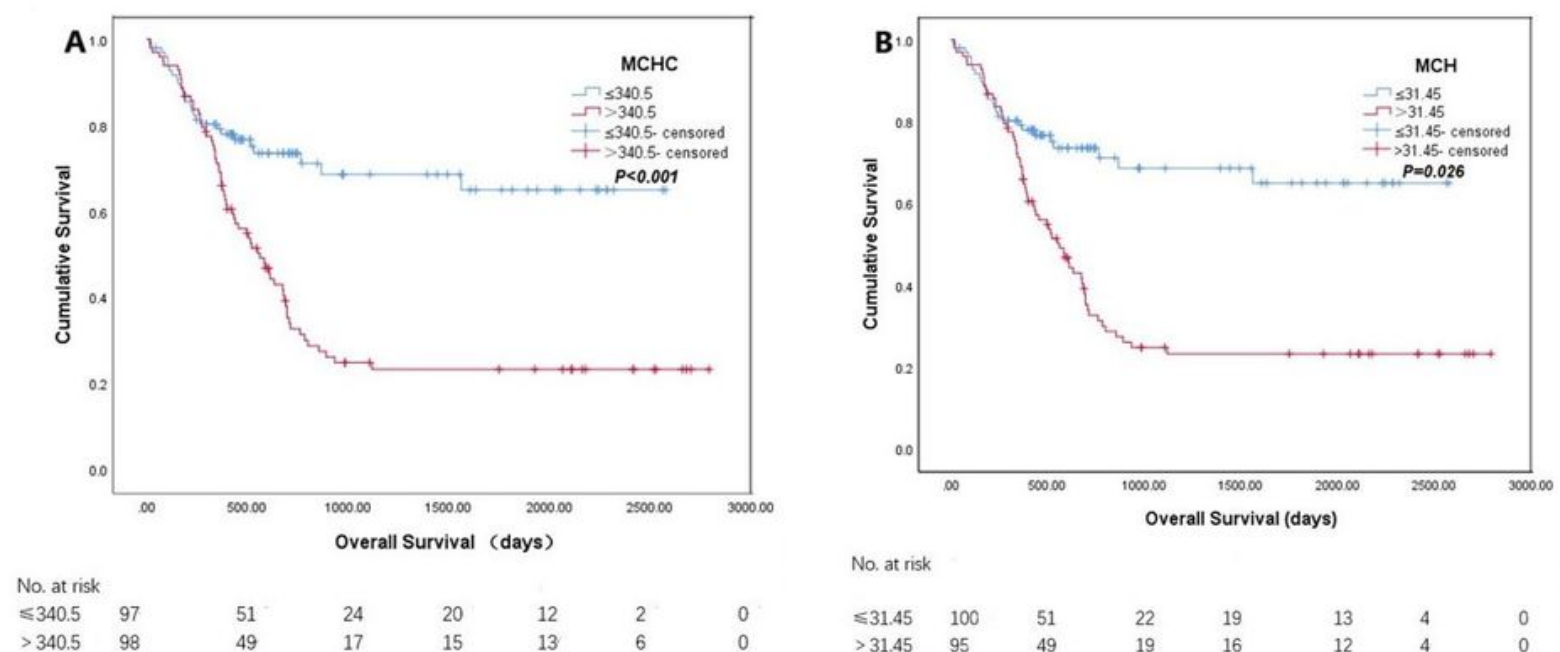

No. at risk
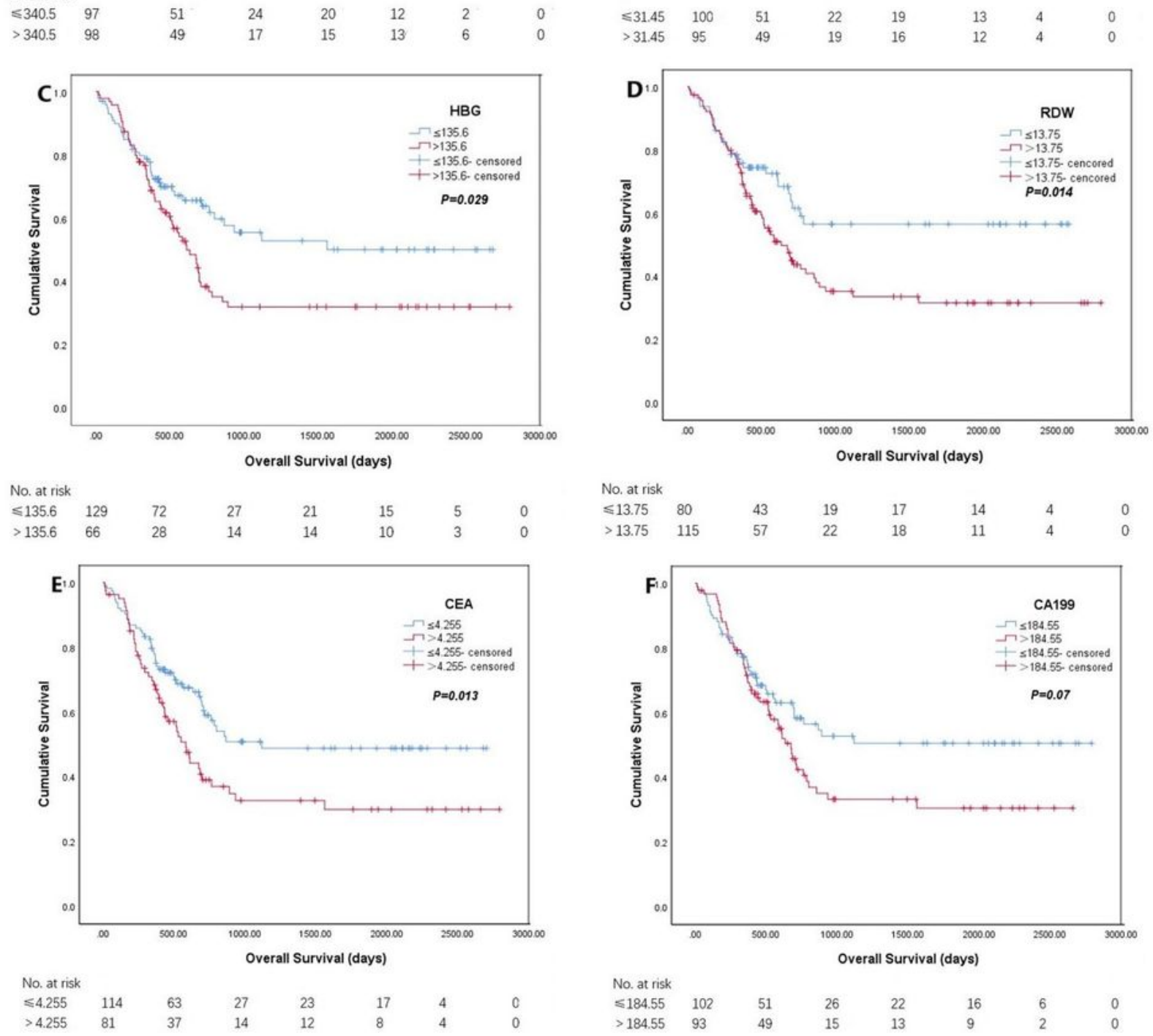

\section{Figure 2}

kaplan-Meier survival curves of resectable PC patients in training set based on $\mathrm{MCHC}, \mathrm{MCH}, \mathrm{HBG}, \mathrm{RDW}$, CEA. (A) Data in training set compares $M C H C \leq 340.5 v s$. $>340.5(p<0.05)$. (B) Data in training set compares $\mathrm{MCH} \leq 31.45 \mathrm{vs} .>31.45(\mathrm{p}<0.05)$. (C) Data in training set compares $H B G \leq 135.6 \mathrm{vs} .>135.6(\mathrm{p}<$ 0.05). (D) Data in training set compares RDW $\leq 13.75 \mathrm{vs} .>13.75(\mathrm{p}<0.05)$. (E) Data in training set compares CEA $\leq 4.255 v s .>4.255(p<0.05)$. 

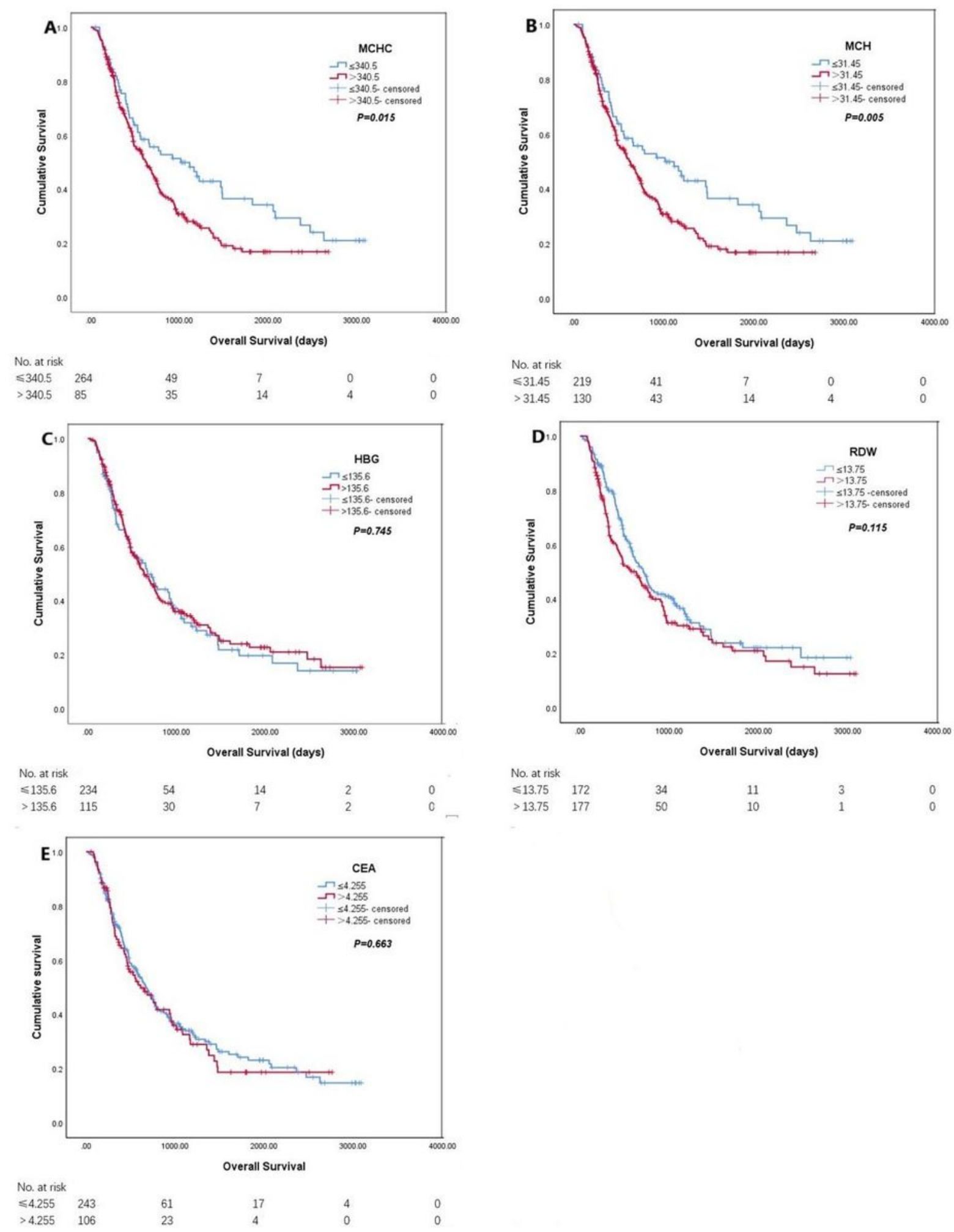

\section{Figure 3}

Kaplan-Meier survival curves of resectable PC patients in validation set based on $\mathrm{MCHC}, \mathrm{MCH}, \mathrm{HBG}, \mathrm{RDW}$, CEA. (A) Data in validation set compares $M C H C \leq 340.5 v s$. $>340.5(p<0.05)$. (B) Data in validation set compares $\mathrm{MCH} \leq 31.45 \mathrm{vs} .>31.45$ ( $\mathrm{p}<0.05$ ). (C) Data in validation set compares HBG $\leq 135.6 \mathrm{vs} .>135.6(\mathrm{p} \otimes$ 0.05). (D) Data in validation set compares RDW $\leq 13.75 \mathrm{vs} .>13.75(\mathrm{p} \otimes 0.05)$. (E) Data in validation set compares CEA $\leq 4.255 \mathrm{vs} .>4.255(\mathrm{p} \otimes 0.05)$. 

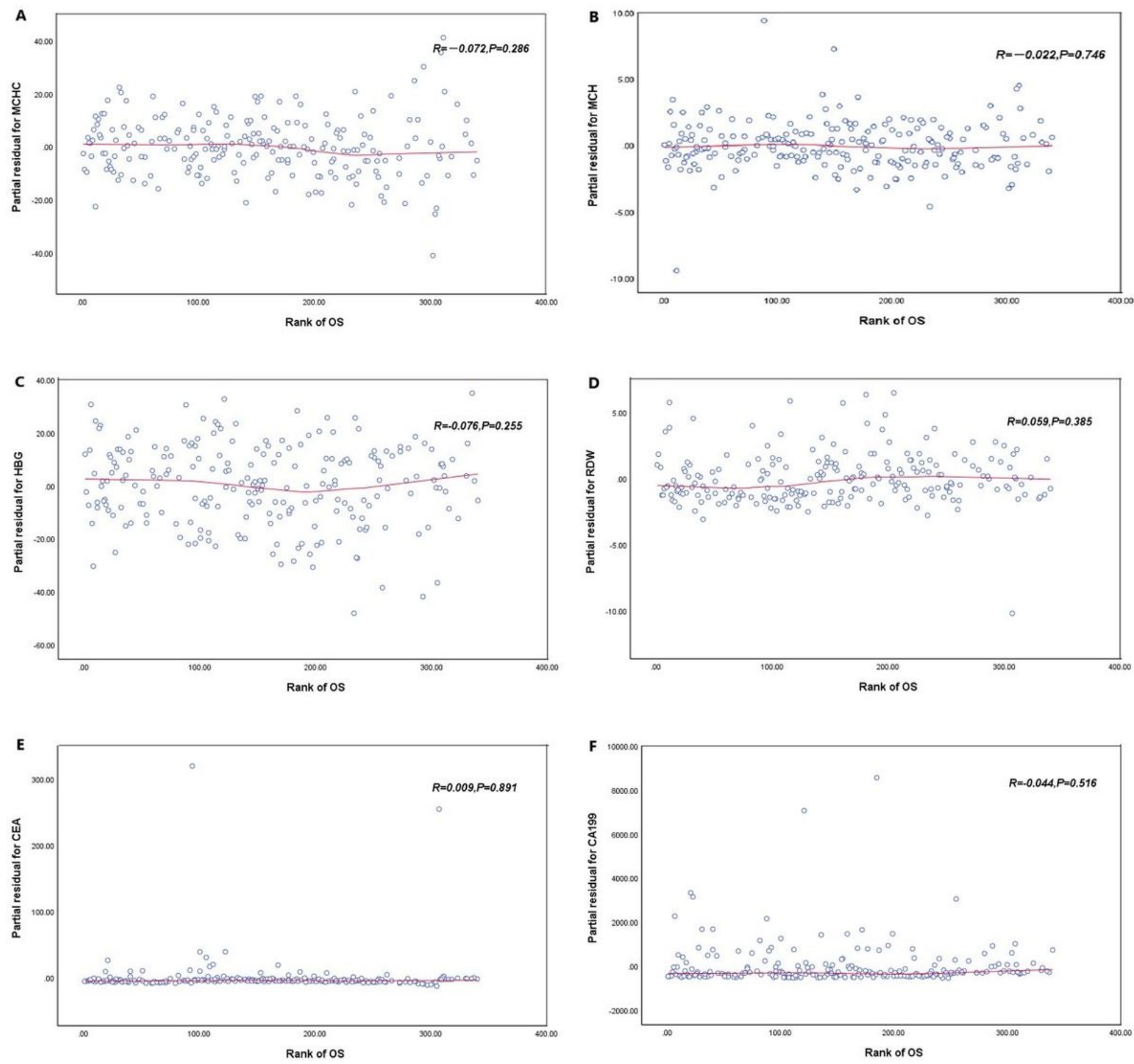

Figure 4

Schoenfeld residual method of resectable PC patients based on MCHC, MCH, HBG, RDW, CEA. (A) Data

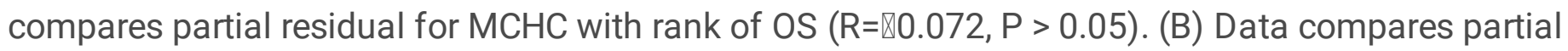
residual for $\mathrm{MCH}$ with rank of OS $(R=\llbracket 0.022, P>0.05)$. (C) Data compares partial residual for HBG with rank of OS $(R=\varangle 0.076, P>0.05)$. (D) Data compares partial residual for RDW with rank of OS $(R=0.059, P$ $>0.05)$. (E) Data compares partial residual for CEA with rank of OS $(R=\llbracket 0.009, P>0.05)$. 